\title{
Substantia nigra pars reticulata neurons code initiation of a serial pattern: implications for natural action sequences and sequential disorders
}

\author{
Melanie Meyer-Luehmann, ${ }^{1,4}$ Jeffrey F. Thompson, ${ }^{2,4}$ Kent C. Berridge ${ }^{3}$ and J. Wayne Aldridge ${ }^{3,4}$ \\ ${ }^{1}$ Department of Neuropathology, University of Basel, Switzerland \\ 2Physiological Science, 4117 LSB, University of California, Los Angeles, Los Angeles, CA 90095-1606, USA \\ ${ }^{3}$ Department of Psychology, University of Michigan, Ann Arbor, MI 48109-1109, USA \\ ${ }^{4}$ Department of Neurology, University of Michigan, Ann Arbor, MI 48109-0489, USA
}

Keywords: basal ganglia, dopamine, fixed action pattern, grooming, Huntington's disease, movement sequences, movement, neuroethology, neuronal activity, obsessive-compulsive disorder, Parkinson's disease, rat, serial order, syntax, Tourette's syndrome

\begin{abstract}
Sequences of movements are initiated abnormally in neurological disorders involving basal ganglia dysfunction, such as Parkinson's disease or Tourette's syndrome. The substantia nigra pars reticulata (SNpr) is one of the two primary output structures of the basal ganglia. However, little is known about how substantia nigra mediates the initiation of normal movement sequences. We studied its role in coding initiation of a sequentially stereotyped but natural movement sequence by recording neuronal activity in SNpr during behavioural performance of 'syntactic grooming chains'. These are rule-governed sequences of up to 25 grooming movements emitted in four predictable (syntactic) phases, which occur spontaneously during grooming behaviour by rats and other rodents. Our results show that neuronal activation in central SNpr codes the onset of this entire rulegoverned sequential pattern of grooming actions, not elemental grooming movements. We conclude that the context of sequential pattern may be more important than the elemental motor parameters in determining SNpr neuronal activation.
\end{abstract}

\section{Introduction}

The substantia nigra pars reticulata (SNpr) is a major output nucleus of the basal ganglia (along with medial globus pallidus). While roles for nigrostriatal projections, neostriatum and globus pallidus in movement and its pathophysiology are well established (Mink, 1996; Wichmann \& DeLong, 1996), fewer studies have focused on the role of SNpr (DeLong et al., 1983; Schultz, 1986a; Gulley et al., 1999; Wichmann et al., 1999). Like medial globus pallidus, SNpr receives fast, divergent, excitatory input from the subthalamic nucleus in addition to slower, convergent inhibitory input from the neostriatum. Cortical information channeled via the striatum reaches SNpr both directly from the neostriatum and indirectly via the lateral globus pallidus and subthalamic nucleus (Albin et al., 1989; Smith et al., 1998).

Correspondence: Dr J. Wayne Aldridge, Departments of Neurology and Psychology, University of Michigan, 300 North Ingalls \#3D03, Ann Arbor, MI 48109-0489, USA

E-mail: jwaynea@umich.edu

${ }^{1}$ Present address: Department of Neuropathology, University of Basel, Switzerland

${ }^{2}$ Present address: Physiological Science, 4117 LSB, University of California, Los Angeles, Los Angeles, CA 90095-1606, USA

${ }^{3}$ Present address: Department of Psychology, University of Michigan, Ann Arbor, MI 48109-1109, USA

${ }^{4}$ Present address: Department of Neurology, University of Michigan, Ann Arbor, MI 48109-0489, USA

Received 14 March 2002, revised 15 July 2002, accepted 5 August 2002
Sequencing of both action and cognition may be important behavioural functions of the basal ganglia (Kermadi \& Joseph, 1995; Mushiake \& Strick, 1995; Beiser \& Houk, 1998; Berns \& Sejnowski, 1998; Schultz, 1998; Waddington et al., 1998; Matsumoto et al., 1999). Although language is the ultimate syntax example, movement sequences besides speech may have syntactic properties too if their serial order is coordinated separately from the individual movements (Lashley, 1951). For example, patients with Parkinson's or Huntington's disease have difficulties in ordered combinations of actions (Harrington \& Haaland, 1991; Curra et al., 1997). Parkinson's patients also have difficulty articulating (Ho et al., 1998) and comprehending speech (Westwater et al., 1998), and have specific deficits in linguistic syntax (Lieberman et al., 1992; Lieberman, 2001) and cognitive shifts (Van Spaendonck et al., 1996). Conversely, initiation of excessively stereotyped sequences is a feature of obsessive-compulsive disorder and Tourette's syndrome (Rapoport \& Wise, 1988; Toates, 1990), which can sometimes involve speech sequences too (O'Quinn \& Thompson., 1980; Lang et al., 1993). Thus, the basal ganglia are implicated in syntactic patterns of action, language and thought (Marsden \& Obeso, 1994; Redgrave et al., 1999; Lieberman, 2001).

Rodent grooming behaviour is useful for neurobehavioural analyses of action syntax because it has natural rule-governed syntactic sequences (Berridge et al., 1987). Previously, we demonstrated striatal participation in natural syntactic grooming chains (Aldridge et al., 1993; Aldridge \& Berridge, 1998). Neurons in the anterior dorsolateral neostriatum activated during syntactic grooming pat- 

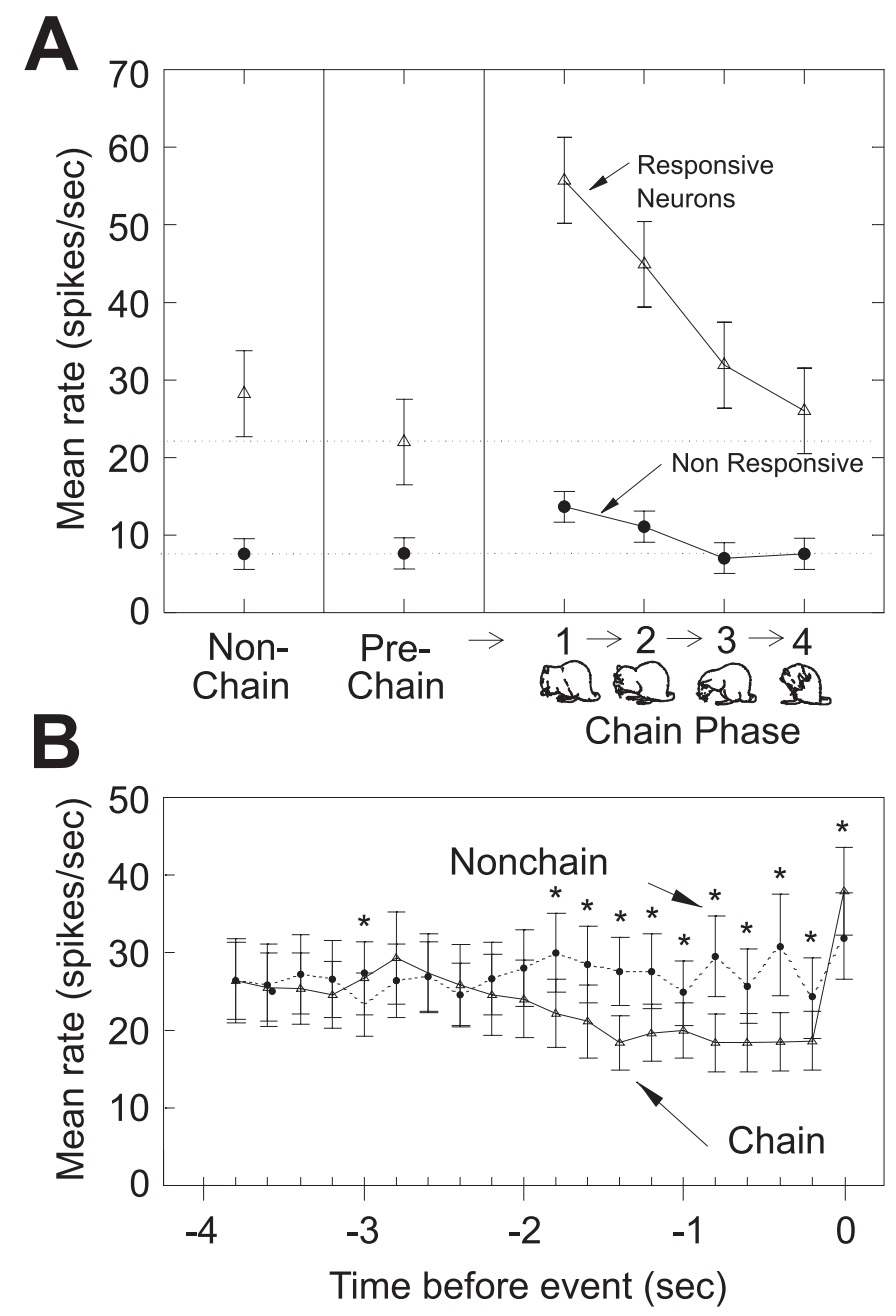

FIG. 1. Mean firing rates. (A) The mean firing rate in spikes/s was computed for (left) nonchain grooming bouts, (middle) prechain baseline and (right) syntactic chain epochs. Responsive neurons ( $\Delta$, top line) and nonresponsive neurons $(\boldsymbol{O}$, lower) are plotted separately. The prechain period served as the baseline for comparison to both syntactic chains and nonchain bouts of grooming actions. Individual phases of the syntactic chain were analysed separately (Phases 1-4). Non-responsive neurons were generally in the ventral regions of the SNpr, fired more slowly, and showed no significant mean changes in firing in any epochs. Responsive neurons in central SNpr regions fired significantly faster during chain than nonchain grooming (ANOVA, $P<0.001, n=25$ ). This comparison includes only neurons that had both syntactic chain and nonchain grooming responses. Phase 1 of syntactic chains had a significantly higher mean firing rate than any of the other phases (ANOVA, $P<0.05, n=25$ ), and firing rates declined significantly after Phase 2. (B) Mean rate for the period $4 \mathrm{~s}$ before the onset of the elliptical stroke in Phase 1 or nonchain grooming in bins $200 \mathrm{~ms}$ wide $(\bullet$, dashed line: nonchain; $\triangle$, solid line: chain). The mean and SEM of each bin is aligned to the end of the bin (e.g. -4.0 to $-3.8 \mathrm{~s}$ ). ${ }^{*} P<0.05$, matched-pairs $t$-test, nonchain vs. chain. Rates deviate consistently beginning in bin -2.0 to $-1.8 \mathrm{~s}$. The last bin $(-0.2$ to $0 \mathrm{~s})$ in each series begins to rise with the onset of movement.

terns, but not when the same movements were emitted in nonchain sequences (more random) suggesting that striatal neurons may code serial order (Aldridge \& Berridge, 1998). Excitotoxin lesions of dorsolateral neostriatum disrupt the serial order of syntactic grooming chains (without disrupting the movements themselves) (Cromwell \& Berridge, 1996). In contrast, lesions of ventromedial neostriatum, motor cortex or cerebellum disrupt grooming movements, but not the serial order of syntactic chain sequences (Berridge \& Whishaw, 1992;
Cromwell \& Berridge, 1996). This double dissociation of sequence vs. movement deficits, plus the electrophysiological coding of sequence, suggests basal ganglia implement the natural sequential pattern rather than merely mediating elemental movements used in it and in other grooming.

The SNpr is a key target of striatal output, and thus a prime candidate to control sequential patterns (Levy et al., 1997; Mahon et al., 2000; Kolomiets et al., 2001). Here we show that SNpr neurons in rats fire during the initiation of syntactic grooming chain patterns, and code aspects of syntactic context also during the remainder of the patterns. We find that SNpr neurons fire differently in syntactic grooming chains than during equivalent grooming movements performed outside the pattern. Thus, central SNpr neurons appear to specifically code the 'syntax' of this grooming sequence.

\section{Materials and methods \\ Overview}

A complete syntactic grooming chain consists of four sequential phases (see Cromwell \& Berridge, 1996; Aldridge \& Berridge, 1998; Berridge \& Aldridge, 2000a; Berridge \& Aldridge, 2000b). Phase 1, lasting approximately $1 \mathrm{~s}$, has five to nine rapid elliptical bilateral strokes over the nose and mystacial vibrissae. Elliptical stroke movements are rare outside syntactic chains. Thus, a series of fast elliptical strokes serves as an excellent marker for the initiation of syntactic chains (see Fig. 2). Phase 2 is characterized by one or two unilateral paw strokes asymmetrically past the region of the mystacial vibrissae. Phase 3 is a series of three to six large-amplitude bilaterally symmetrical strokes aimed above the eyes, often at the top of the head behind the ears, and performed synchronously and usually symmetrically by the two paws. Phase 4 starts with an initial postural turn and head lowering followed by body licking over the lateral and ventral torso.

\section{Animals}

Sprague-Dawley male rats $(250-400 \mathrm{~g})$ were used for these experiments and were housed on a 12-h light : 12-h dark schedule with the lights out at $11.00 \mathrm{~h}$ each day. The active part of the rat's circadian cycle (the dark period) coincided with our recording times (early afternoon). To habituate the rats to the recording environment they were handled and placed in the recording chamber on 3 days prior to surgery for $\approx 20 \mathrm{~min}$ each time. The University of Michigan Unit for Laboratory Animal Medicine approved all procedures.

\section{Surgery}

Aseptic surgical preparation occurred 3-5 days before recording under general anaesthesia (ketamine $100 \mathrm{mg} / \mathrm{kg}$, xylazine $10 \mathrm{mg} / \mathrm{kg}$, both i.p.) Multiple electrode arrays (eight wires; Jaeger et al., 1990) were placed in the SNpr (centred around AP $-1.9, \mathrm{ML} \pm 3.5$ and DV $-5.4 \mathrm{~mm}$ with respect to bregma).

\section{Behavioural and neurophysiological recording}

For recording, rats were placed in a box $(28 \times 35 \mathrm{~cm})$ over a glass floor under which a mirror was positioned to reflect a view of the head and body of the rat into the lens of the video camera. Recorded single neuron activity was amplified, bandpass filtered with cutoffs set to 100 and $10000 \mathrm{~Hz}$ and recorded on a computer (DataWave Technologies, Longmount, CO 80501). Waveform characteristics were viewed on an oscilloscope and spike discharge was monitored by audio amplifier. Behavioural activity was video taped simultaneously along with time information on each frame synchronized to 


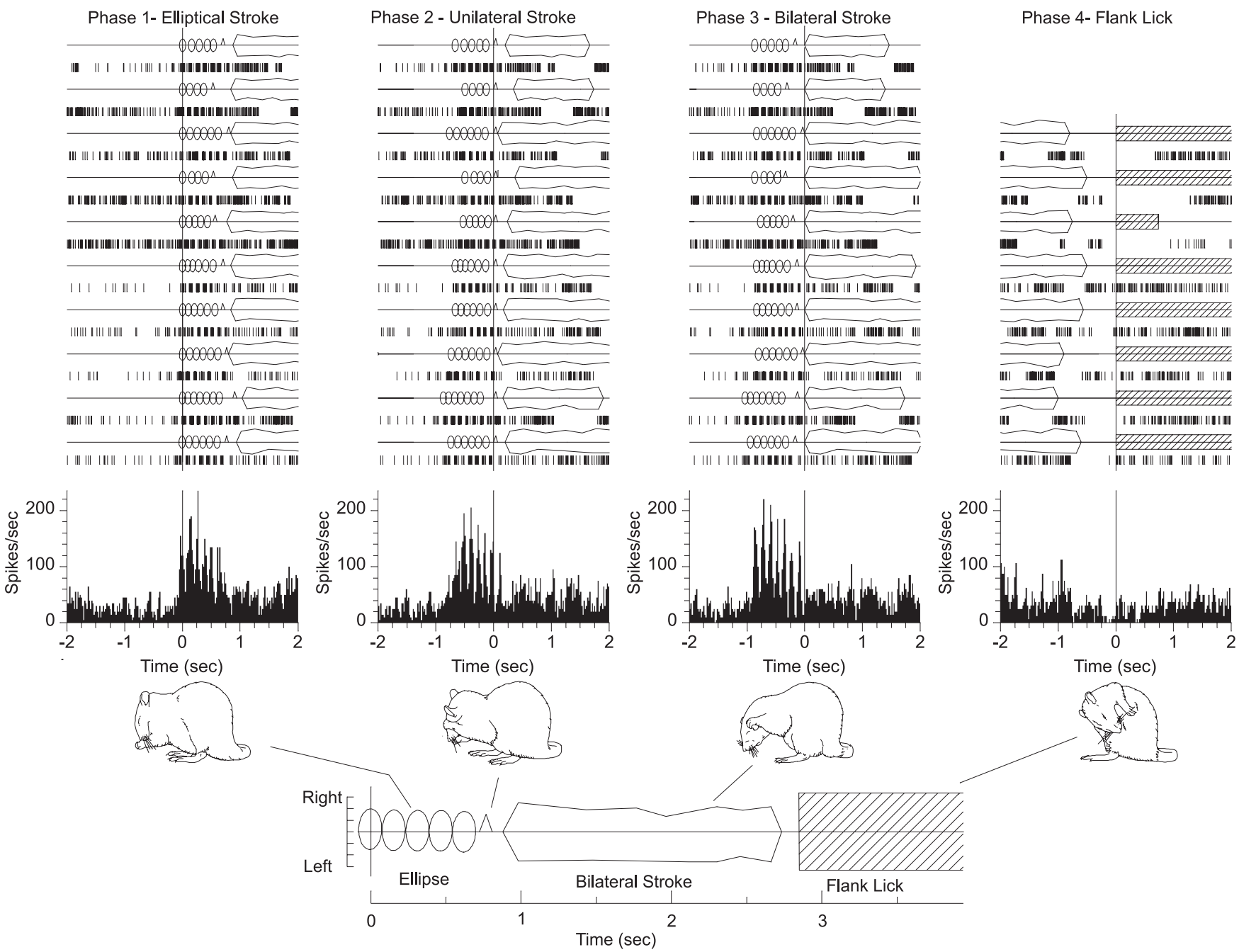

FIG. 2. Neuronal coding of syntactic grooming phases in central SNpr cells. Rasters show individual spike trains from a single neuron along with perievent time histograms indicating the average firing rate (y-axis) in bins $20 \mathrm{~ms}$ wide. Time 0 in each histogram and raster is defined as the onset of the first stroke defining that phase. The symbolic choreographic notation of grooming movements inset at the bottom illustrates the amplitude of medial-lateral paw movements across the face from the midline as a function of time. In each raster, the choreographic diagram above each spike train shows the timing of the movements for the associated chain. Dashed lines indicate nonchain grooming, which has more random sequences of grooming actions. As was typical of most neurons in central SNpr that coded sequence initiation, an increase in activity was associated with the onset of Phase 1 (elliptical strokes about the nose and mouth). This activation continued during Phase 2 and then declined during Phases 3 and 4 of the chain.

neuronal recording clock. Grooming, walking, periods of quiet resting and other movements were emitted spontaneously during a 2-h session.

\section{Behavioural analysis}

A frame-by-frame analysis of the videotapes was conducted off-line using a computer-assisted behavioural notation program developed in this lab. This program extracts the time code from a video frame selected, identified by the experimenter as representing the onset of an individual action. In a frame-by-frame inspection of the tape with a slow-motion and single-frame-advance VCR, the onset time information associated with each action was stored in a database of limb and body movements (e.g. rearing, stepping, head turning). All bouts of grooming were demarcated completely. A cessation of grooming for $\geqslant 5 \mathrm{~s}$ defined the end of a bout. Breaks of $<5 \mathrm{~s}$ were deemed contiguous grooming for this analysis. The onsets and ends of all grooming actions within bouts having syntactic chains were identified, and the analysis included complete descriptions of 'syntactic grooming chains' (sequentially stereotyped 4-phase pat- tern). 'Nonchain grooming' (similar grooming movements emitted in more random orders outside syntactic chains) was sampled in detail to obtain, whenever possible, 10 movements matched to movements of the chain sequence (Fig. 3). Overall, nonchain grooming was more abundant than the chain sequence grooming, because syntactic chains last at most a few seconds and are usually embedded within longer nonchain grooming bouts. For the purpose of quantifying and comparing limb movements across the two types of grooming, some strokes were classified by trajectory amplitude and paw laterality.

\section{Neuronal activity analysis}

We used the programs Stranger (Biographics, Inc., Winston Salem, NC, USA) and Nex (Plexon, Inc., Dallas, TX 75206, USA) to extract firing rate information from spike trains for quantitative comparisons and to construct perievent histograms and rasters around the onsets of grooming actions. Statistical comparisons were made with (Systat, SPSS Inc., Chicago, Illinois 60606, USA).

As a first step, we evaluated the possibility that neurons might show overall rate-coding differences in their spike trains between 


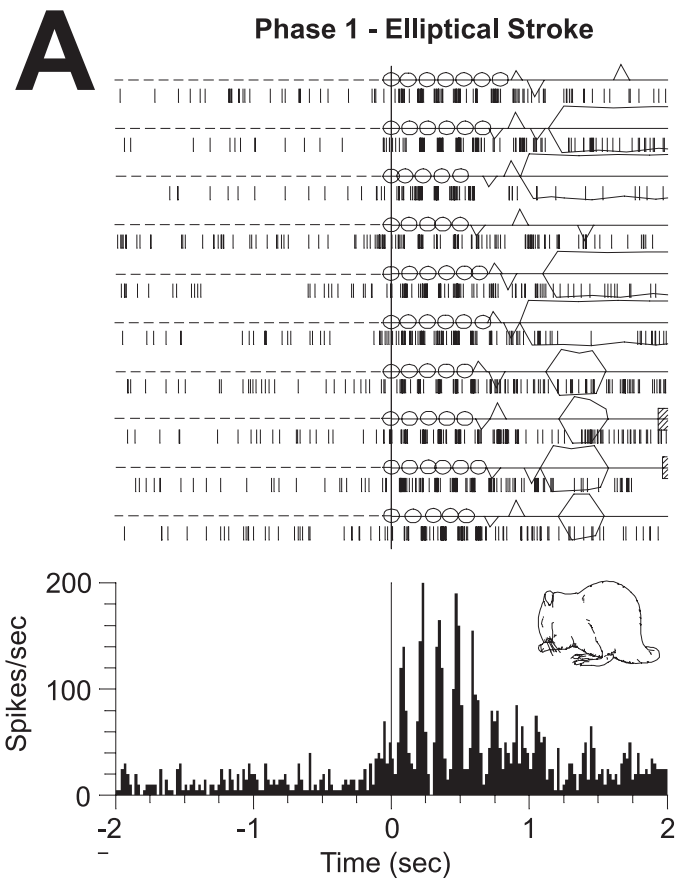

B

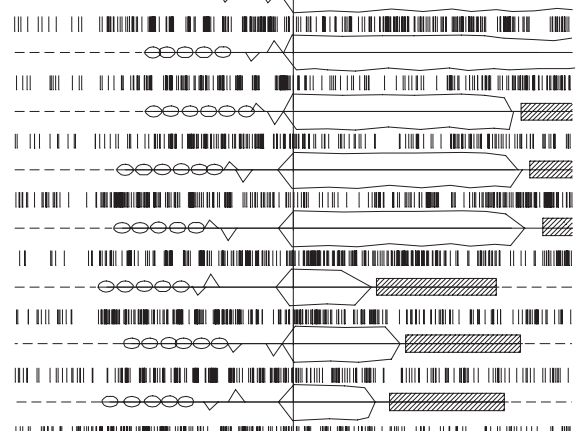

||||| |||| | | ||||||||||||||||||||||||||||||||-|||||||||||||||||||||||||||||||||||||||||||||||| || |||| || || || |||||||| | ||||

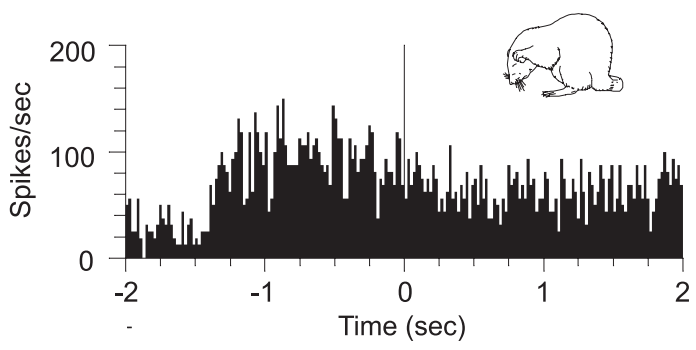

Nonchain Elliptical Stroke
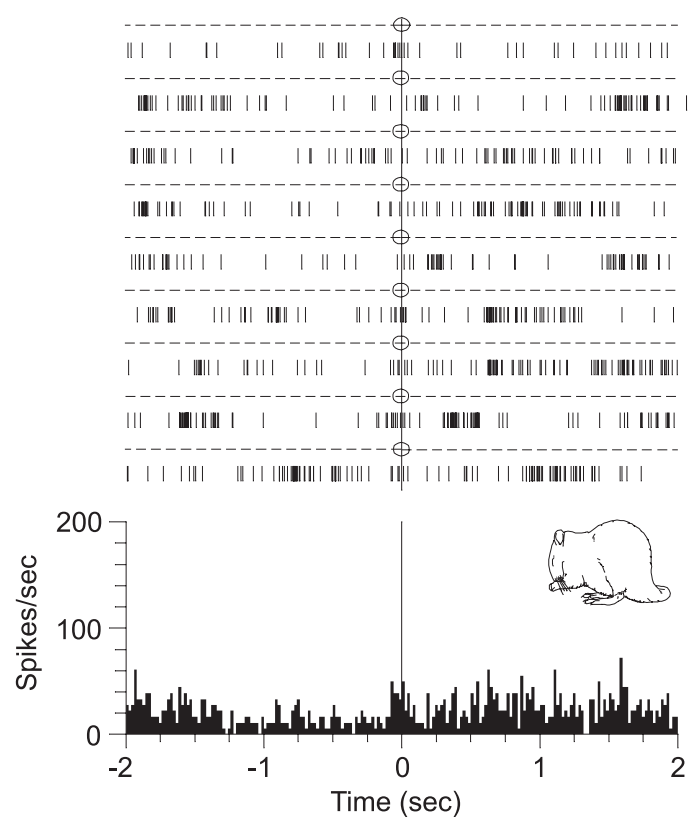

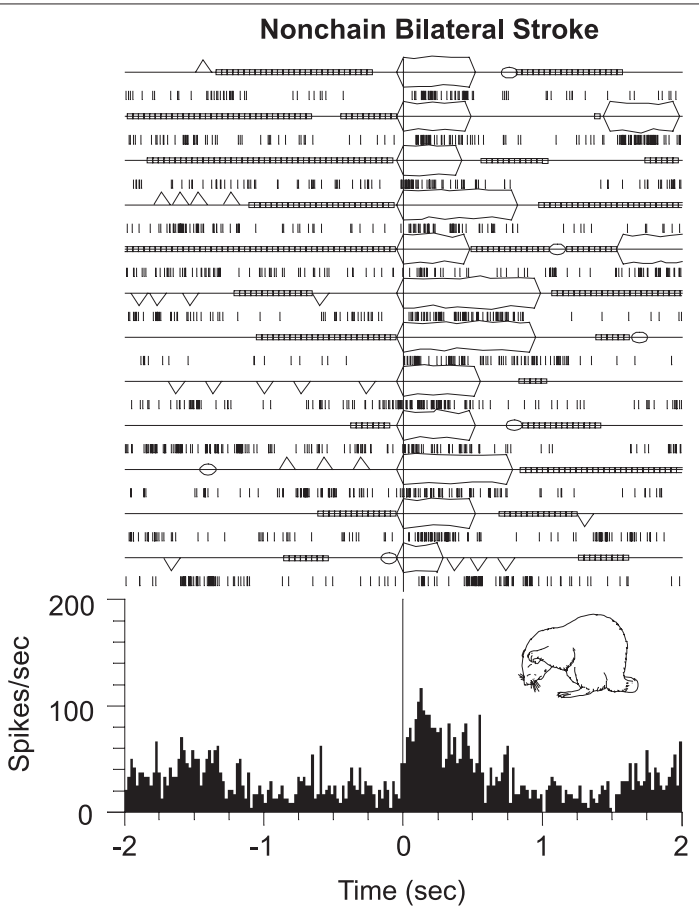

FIG. 3. Sequence dependent firing in SNpr neurons during grooming behaviour. The format of the rasters and histograms is the same as in Fig. 2. The choreography insets in each raster line show the timing of the strokes and in the case of chains, the preceding and following actions. Dashed lines indicate nonchain grooming that has random mixtures of grooming actions. (A) Neuronal firing at the onset of syntactic grooming chains was more vigorous than firing during nonchain motor equivalents to Phase 1 strokes (i.e. during small bilateral nose strokes performed in more random grooming sequences). (B) Neuronal

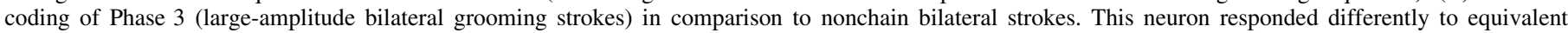
movements depending on whether they occurred in (left) chain or in (right) nonchain grooming. During Phase 3 of syntactic chains (left), there was little relative change in activity at time 0 . During nonchain bilateral strokes (right), however, there was a clear increase in firing. Note that that neuronal activity

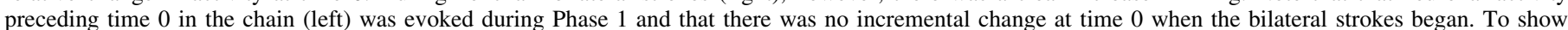
that absence of sequence in nonchain grooming, the choreography plots for nonchain bilateral strokes (right) have all of the strokes before and after the onset of the bilateral stroke marked. The choreography format is the same as in Fig. 2 with the addition of connected open squares to show paw-licking movements.

syntactic chain sequences and nonchain grooming. This comparison was accomplished by computing the average firing rates in behavioural epochs demarcated by the onsets and ends of nonchain grooming and syntactic chain phases along with a period from 2 to
$1 \mathrm{~s}$ before Phase 1 of syntactic chain. The latter period was selected as a background baseline for comparisons to provide the most consistent behavioural state for a general comparison. Behaviourally, this period consisted of a mixture of nonchain grooming actions 

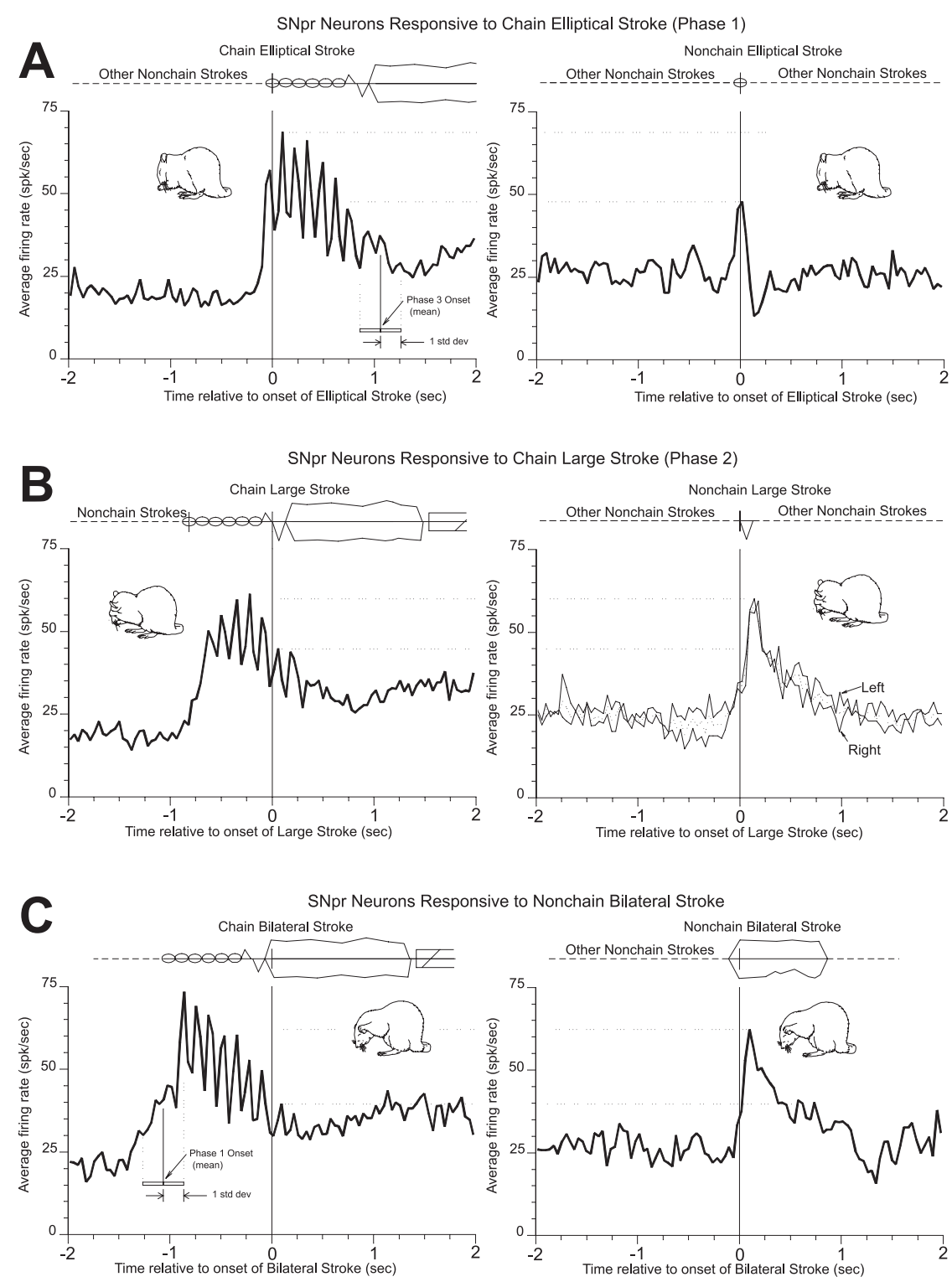

FIG. 4. Comparisons of peak neuronal activation. Perievent time histograms for responsive neurons were averaged for both (left) chain and (right) nonchain grooming. The choreographic insets portray the grooming movements with dashed lines to indicate variable mixtures of nonchain grooming. Dotted lines on the graph depict the peak activation levels during chain and nonchain grooming. These histograms represent averages of many movements over several cells and animals. Events apart from the one at the alignment point (time 0) are smeared in time due to the variability of relative movements with respect to each other. (A) Neurons responsive to onset of the elliptical stroke reached higher peaks than during the nonchain ellipse movement. In nonchain grooming, ellipse movement occurred singly in contrast to chains with 5 or 6 movements. For reference, the average onset of Phase 3 at $1.06 \pm 0.20 \mathrm{~s}(\mathrm{mean} \pm \mathrm{SD})$ after Phase 1 is marked by the inset. Firing rate declines during Phases 1 and 2 of the chain. (B) Neurons responsive to chain Phase 2, unilateral stroke were compared with their nonchain counterparts. Activation was greater during nonchain grooming. There was no apparent laterality effect as illustrated by separate lines for averaged left and right movements right (nonchain diagram). (C) Neurons responsive to nonchain bilateral strokes were averaged for this comparison to the same movements in chains. The inset marks the average onset of Phase 1 and its associated variability. This smearing in time of the onset of Phase 1 relative to the alignment point at time 0 accounts for the less abrupt increase in activation at that time compared to the diagram in A.

because syntactic grooming chains are almost invariably embedded within bouts of nonchain grooming (Berridge et al., 1987). The baseline period was considered to end $1 \mathrm{~s}$ before the first movement of the chain to allow separate detection of any preparatory, prechain changes in activation. The rates in these epochs were compared statistically across the entire sample of responsive and nonresponsive neurons.

For fine-grained analyses of dynamic neuronal activity, we constructed and evaluated perievent histograms and rasters by visual inspection and quantitative comparisons (Churchward et al., 1997). Excitations were defined as increases $>150 \%$ of the baseline period before the chain and inhibitions were defined as decreases $(<50 \%$ baseline). In some cases, changes might be superimposed on ongoing neuronal activation if there was an earlier movement in a sequence (e.g. unilateral strokes of Phase 2 or 3 following activation of Phase 1). To be considered responsive, excitations or inhibitions identified in the perievent histogram had to be associated with concomitant changes of activity with the same direction and time course in more than half the individual trials of the corresponding raster. For quantitative comparisons between neurons, we computed the average firing rates in a $200-\mathrm{ms}$ bin following each behavioural action from the perievent time histograms. 

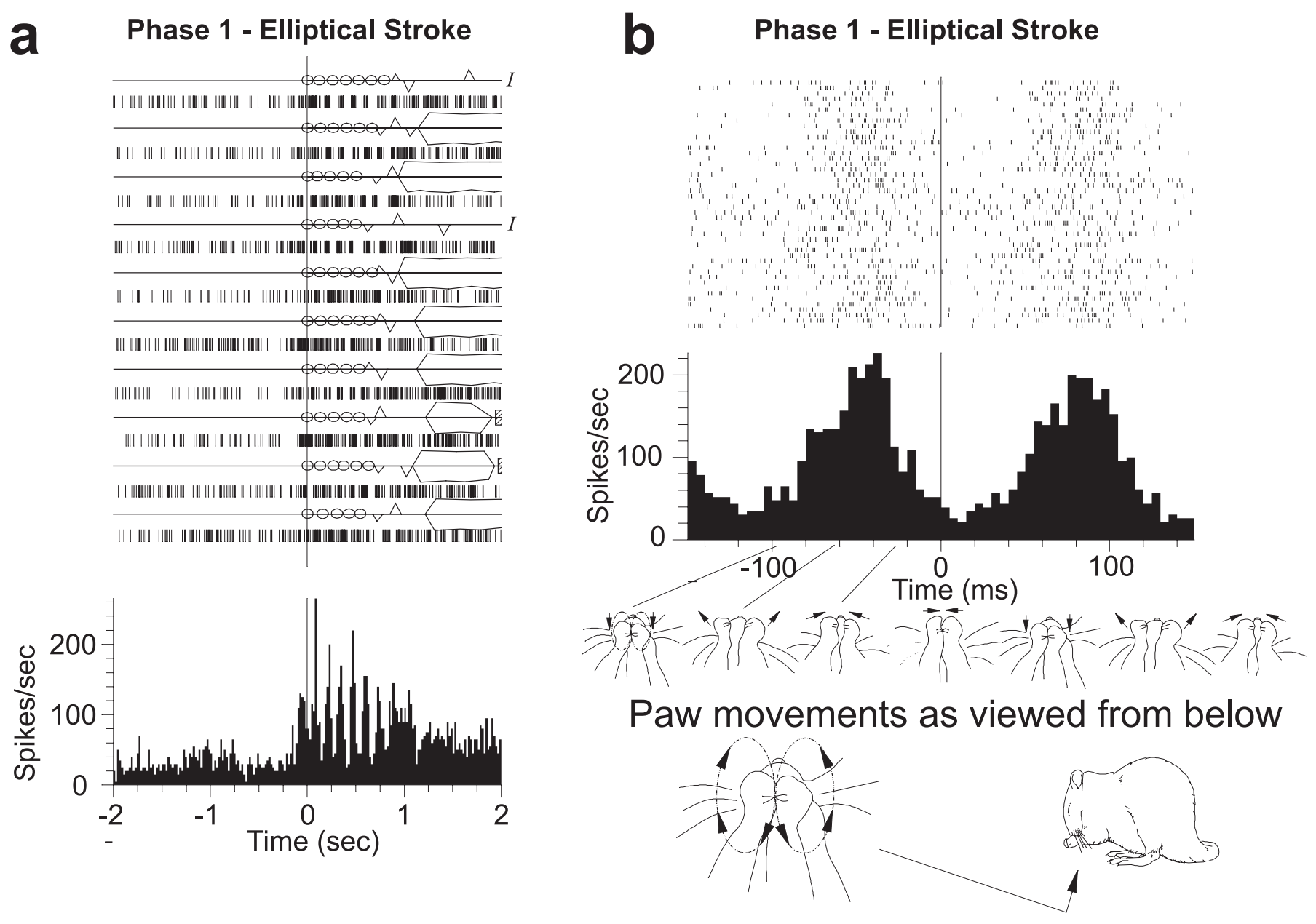

Fig. 5. Fine structure of activation pattern at onset of syntactic chains. (A) The neuron fired 5-6 repeated phasic activations during the 5-6 elliptical strokes of syntactic Phase 1. (B) The perievent time histogram and raster on the right shows activity of the same neuron aligned with a shorter time axis encompassing only two elliptical strokes within Phase 1. The drawings (tracings from video tape frames) below the $x$-axis portray the movement of the rat's paws around the mouth at the indicated time points during the two strokes. The arrows indicate the direction of movement. Increased firing occurs with the forward-directed movement along the outside of the ellipse from the midline to the front of the nose. A silent period occurs as the paws join in front of the nose, and continues during the backward part of the stroke as the paws are drawn behind the chin. The cycle then repeats. For visual clarity, time zero was defined as the instant the paws joined in front of the nose in each ellipse. That time point in the first ellipse of the sequence defines the onset of Phase 1. Neuronal activity during Phase 1 actually followed the onset of ellipse stroke movement, because the forward movement of the paws had already occurred by the time the paws met in front of the nose.

Mean values are quoted \pm SEM unless otherwise specified.

\section{Histology and localization of recording site}

At the end of the recording session, we made an electrolytic lesion to mark the location of the electrode. Animals were killed with an overdose of pentobarbital and perfused transcardially with phosphatebuffered saline followed by $10 \%$ formalin in $0.9 \%$ phosphatebuffered saline. Brains were sectioned $(40 \mu \mathrm{m} / \mathrm{section})$ and stained with Cresyl violet. Recording sites were identified microscopically and mapped on a standard atlas (Swanson, 1992). Neuronal firing properties in addition to the histological localization confirmed the identity of SNpr neurons included in this analysis.

\section{Results}

Data were recorded, in 11 rats, from 47 neurons that were histologically verified to be in SNpr. Over half of SNpr neurons $(55 \%, n=26)$ were responsive during grooming. All but one of these neurons were responsive during both chain and nonchain grooming, although neural activity typically differed in these two contexts as described below. Grooming-responsive cells were generally located centrally within the SNpr. A second group of neurons $(45 \%, n=21)$ did not respond during any grooming behaviour and tended to be located more ventrally in the sampled SNpr regions. Responsive neurons had irregular activity patterns and large-amplitude, brief action potential waveforms characteristic of $\mathrm{SNpr}$ and pallidal neurons (Schultz, 1986a). All recording sites were ventral to the pars compacta (dopamine cell) region in dorsal SNpr. No dopaminergic-like patterns of cell activity (Schultz, 1986b) or waveforms were observed, which is consistent with a conclusion that we sampled neurons in SNpr rather than dopamine neurons in pars compacta.

\section{Population rate coding of syntactic chain grooming}

We compared overall firing rates during syntactic grooming chains (sequentially stereotyped 4-phase pattern) with nonchain grooming (similar grooming movements emitted in more random orders outside syntactic chains; Fig. 1) to test the hypothesis that overall firing rates 
might code the serial pattern of chain sequences. We observed a strong modulation in certain phases of the chain in neurons located in the central region of SNpr. Initial analysis showed that firing rates of neurons in central SNpr increased significantly during syntactic grooming chains compared to both random nonchain sequences of grooming behaviour and the prechain baseline (Fig. 1; ANOVA $P<0.001, n=25)$. Further post hoc analysis of firing rate during each specific phase of syntactic chains (Phases 1-4) showed that Phase 1 specifically had a significantly higher mean firing rate than all the other phases (Fig. 1; ANOVA $P<0.05, n=25$ ). Other neurons in more ventral portions of SNpr exhibited no change in mean firing rate during any grooming period and had slower overall firing rates (Fig. 1). Those nonresponsive neurons were not analysed further.

Among SNpr neurons that were responsive to grooming behaviour, we detected a small (22\%) but significant and persistent decrease in activity in the baseline period -1.8 to $-0.2 \mathrm{~s}$ before syntactic sequences began (Fig. 1B). The animals were almost invariably engaged in nonchain grooming at that time, and so a prechain depression of firing during an ongoing grooming bout does not indicate a corresponding pause in grooming or absence of movement, nor was there any other visible change in grooming movements during that period compared to preceding movements. Therefore, this prechain neuronal depression may indicate an anticipatory inhibitory signal that predicts or prepares for an impending syntactic chain (see below). The mean $( \pm$ SEM) firing rate during the prechain baseline period was $22.0 \pm 3.9$ spikes/s, compared to $28.3 \pm 4.8$ during nonchain grooming $(P<0.001$, matched-pair $t$-test). In contrast to this anticipatory suppression prior to syntactic chains, the periods preceding various nonchain strokes (elliptical, unilateral or bilateral paw strokes $)$ did not differ from each other $(P=0.522$, Friedman two-way ANOVA; see Fig. 4).

\section{Dynamic neural coding}

For the fine-grained evaluation of dynamic neuronal coding in individual neurons, perievent time histograms and rasters were constructed about the onsets of grooming actions in both chain (syntactic patterns) and nonchain (nonsyntactic) contexts. Neurons in central SNpr had clear activity changes associated with the onset of syntactic grooming chains (Fig. 2). Excitations were more common (74\% of responsive cells had only excitation). Inhibitory responses were less frequent ( $23 \%$ of responsive cells) and always occurred in neurons that had excitatory responses as well.

SNpr neurons appeared to be preferentially responsive to the onset of the syntactic sequence. Most neurons (73\%, 19 of 26 responsive neurons) were responsive only during the first two phases of the syntactic sequence. Neuronal activity declined from the end of the first phase of the chain over subsequent phases (Fig. 2). In 22 neurons with activity in both Phase 1 and Phase 2, the rate was significantly higher in Phase 1 than Phase $2(50.4 \pm 8.1$ vs. $41.5 \pm 6.7$ spikes/s, respectively; $P=0.049$, matched-pairs $t$-test). The onset of Phase 1 was marked by the most vigorous spike activity that we observed during syntactic chains, with values ranging from 44 to $494 \%$ higher (average 180\%) than baseline. In contrast to Phases 1 and 2, changes in neuronal activity during Phases 3 and 4 of the syntactic chain were less frequent ( $n=3$ and 6,12 and 23\%, respectively). When they occurred, their peak amplitudes were less than for Phases 1 and 2 and, like Phase 2, they were superimposed on the slow decline in activity from Phase 1 . Other Phase 3 changes in activity were inhibitory ( $23 \%$ of responsive neurons). On average, by Phase 3 , only $12 \%$ of the neurons had rates that exceeded prechain baselines.

\section{Sequential pattern vs. movement coding}

To determine whether neurons were simply coding movements or alternatively coding syntax, we compared quantitatively neuronal activity associated with the onset of movements in syntactic chains to the onset of equivalent grooming movements in nonchain (unpredictable) patterns. The chosen movements in nonchain grooming are morphologically similar to their chain equivalents, but differ principally by the fact that their serial order is unpredictable (Phase 1 equivalents = bilateral paw strokes around the nose or mouth; Phase 2 equivalents = unilateral paw strokes below the eye; Phase 3 equivalents $=$ bilateral paw strokes above the eye; Phase 4 equivalents $=$ postural shift of head towards flank followed immediately by bout of flank licking). The essential difference between chain movements and nonchain movements is the context of serial order. Neurons that code movements should respond in a similar manner to motor equivalents regardless of their sequential order. By contrast, neurons that code sequence properties should respond differently to movements in a syntactic chain compared to equivalent movements in the unpredictable order of nonchain grooming, even though the movements themselves are similar.

We found that more neurons were activated during syntactic chains than during equivalent nonchain grooming movements. Furthermore, activation strength of particular neurons differed in these two contexts even though the morphology of the movements was similar. For example, many neurons (36\%) responsive during Phase 1 did not respond at all or else responded with rate changes much lower than during similar chain grooming movements (Fig. 3A). Neurons which were activated during both syntactic chain and nonsyntactic ellipse movements $(63 \%)$ had quantitatively different responses in the two contexts (Fig. 4A). These neurons had significantly higher firing rates during chain than nonchain grooming (50.1 \pm 8.1 vs. $28.0 \pm 4.6$ spikes/s for chain and nonchain, respectively; $P=0.02$, ANOVA). As noted above, the highest observed firing rates occurred during Phase 1 elliptical strokes at the onset of the grooming sequence.

During unilateral strokes of Phase 2, activation levels declined relative to Phase 1 (e.g. Fig. 2). Like elliptical strokes, the sequence/ nonsequence context of the grooming actions had a potent influence over the neuronal firing even though the morphology of the movements is similar. In contrast to Phase 1 , however, firing rates were significantly faster during nonchain unilateral strokes than during equivalent movements of chain grooming (Fig. 4B; $34.7 \pm 4.4$ vs. $48.5 \pm 5.9$ spikes/s for chain and nonchain, respectively; $P=0.027$, ANOVA). In other words, although neurons responded preferentially to the onset of Phase 1 , once the chain entered later phases they became relatively suppressed compared both to Phase 1 and to nonchain motor equivalents. The context of the syntactic chain pattern caused neurons to be relatively excited during initiation of the pattern but relatively inhibited during terminal phases. There was no significant laterality effect, i.e. no difference between left and right limb movements ( $P=0.7$, ANOvA; Fig. 4B), signifying the context of sequence as opposed to movement parameters as the crucial determinant for activation.

A comparison of the bilateral strokes of Phase 3 to their nonchain motor equivalents extended the conclusion that neuronal responses were suppressed during late phases of syntactic chains. These large synchronized bilateral strokes over the eyes are morphologically nearly identical in the two contexts (Aldridge \& Berridge, 1998). As with Phase 2 vs. nonchain unilateral strokes, the firing rates were significantly higher during nonchain grooming equivalents to Phase 3 (Fig. 4C; $36.6 \pm 5.6$ vs. $58.5 \pm 9.0$ spikes/s for chain and nonchain, 
respectively; $P=0.023$, ANOVA). Interestingly, the bilateral strokes during Phase 3 of the syntactic chain evoked activity from fewer neurons $(12 \%)$ than similar strokes during nonchain grooming $(65 \%$ of neurons; Fig. 3B). Thus, despite the great similarity of the movements in these two contexts, SNpr neurons were markedly activated only by large-amplitude bilateral paw strokes during nonchain grooming sequences, but not by the same movement during Phase 3 of syntactic chains. This preferential coding of nonchain large bilateral paw strokes was the opposite coding pattern seen in Phase 1 , in which the syntactic chain form of equivalent movements was preferentially coded. Finally, a close examination of other strokes that co-occur with nonchain bilateral strokes (Fig. 3B) reveals no apparent high order pattern or Markov influence (Berridge et al., 1987) which might explain the marked activation. Thus, SNpr neurons preferentially coded the onset of the programmed sequence (Phase 1), and then ignored motor parameters during Phase 2 and Phase 3 which ordinarily would have activated them.

As a partial caveat, we note that activity preceding Phases 2 and 3 of syntactic chains were typically different from activity before their nonchain equivalent movements. This occurred because the SNpr excitation caused by Phase 1 did not fully decay before Phase 2 or Phase 3 and so activity preceding late syntactic chain phases was higher than activity preceding nonchain unilateral and bilateral strokes. However, this does not imply that the late-phase suppression was simply an artifact of baselines. Instead, a clear suppression of SNpr neurons during syntactic Phases 2 and 3 was still evident in two ways. First, there was at least a $25 \%$ relative decline in firing within syntactic chains from Phase 1 to Phase 3, and neurons did not maintain their high Phase 1 firing rates in later phases (which they should have done if their response to Phase 3 movements were not suppressed). Most important, absolute firing rates were $\approx 50 \%$ higher during nonchain occurrences of large bilateral grooming strokes than during their equivalent Phase 3 strokes, as noted above. These combined observations indicate that syntactic chains involved a real suppression of SNpr responses to movements during late phases, independent of the difference between preceding baselines.

\section{Neuronal pattern at sequence onset}

Neural activity reached peak rates during the first phase of the chain sequence with some cells firing at 200 spikes/s for brief periods (Fig. 5). During Phase 1, spike trains also had a characteristic temporal pattern consisting of repeated short firing bursts (75$100 \mathrm{~ms}$ ) alternating with pauses $(50-100 \mathrm{~ms})$. These bursts occurred five or six times during Phase 1 , with one burst of spikes associated with each of the five or six elliptical paw stroke movements (Fig. 5). The pauses between bursts coincided with the point in time when the paws joined in front of the nose during every elliptical stroke in the sequence. This was resolved by a frame-by-frame video analysis and plotting the perievent histograms with a time span comparable to the duration of individual strokes of the movement trajectory (Fig. 5B). The relative neuronal silence continued between each Phase 1 paw stroke as the paws moved down from the nose along the midline of the mouth, and then reversed into a burst of rapid spike activity when both paws reversed to move forward along the lateral ventral cheeks, retracing their elliptical trajectory toward the front of the nose. Overall, these repeated bursts of SNpr activity marked the onset of the serial pattern of a whole syntactic chain in a distinct fashion.

Although elliptical strokes signify the onset of the grooming sequence, they rarely represent a 'first' movement in a grooming bout. This was confirmed by carefully examining syntactic grooming with respect to nonchain grooming. Chain grooming sequences occur within continuous bouts of nonchain grooming and they were hardly ever first in a bout of grooming. In addition, chains rarely occurred in isolation. These points are illustrated by the grooming bouts that comprise Fig. 3. The order in the bout in which the first elliptical strokes of Phase 1 occurred varied from the 7 th to the 22 nd (median 12th) in grooming sequence bouts. This was significantly later in sequential order ( $t$-test, $P=0.03$ ) than nonchain elliptical strokes, which varied from the 2 nd to the 27 th (median 7 th). No chains were ever first in a grooming bout. Thus, it is unlikely that the vigorous activation at the onset of a chain represents 'onset of movement' in its simplest form. The critical determinant for neuronal activation with elliptical strokes seemed to be that they were the first movements of the syntactic chain. Phase 1 neurons were not simply coding movement onset or being 'first' in general, but instead seemed to specifically code the initiation of this syntactic sequence of grooming movements.

\section{Discussion}

Our findings demonstrate that neurons in the central pars reticulata portion of the substantia nigra ( $\mathrm{SNpr}$ ) were activated vigorously by grooming movements. The most robust firing occurred during the initiation of syntactic grooming chains with each well-synchronized burst 'winding-up' with each stroke to reach the highest levels. By comparisons of similar grooming movements in syntactic grooming chains vs. more loosely organized nonchain grooming bouts, we found that sequential context was an important determinant of cell activation. Whether movements occurred inside or outside syntactic chains may be even more important than the particular movements themselves in influencing neuronal activation. In contrast to the vigorous activation at the onset of the syntactic grooming chain, later movements of the sequence evoked more activity when they occurred outside the chain in unpredictable strings of nonchain motor actions. Our findings suggest that SNpr neurons may specifically code some aspect of the initiation of this stereotyped grooming sequence, as most neurons were active over the first two phases of the chain sequence and inactive or less active during the last two phases.

Most central SNpr neurons seemed not related at all to the amplitude of individual movements. For example, many neurons fired during small-amplitude movements during Phase 1 of syntactic chains in which paw strokes around the snout stay below the tip of the nose. Yet the same neurons did not fire during larger amplitude movements in Phase 3 of syntactic chains, in which paw strokes typically ascend above the eye, even though both movements involve bilateral upwards extension of the paws, followed by downwards retraction, and the amplitude of Phase 3 movements is much larger. Finally, even neurons that were activated by equivalent amplitude movements in syntactic chains and in nonchain grooming were found upon close inspection to have different responses in the two sequential contexts (e.g. Phase 1 strokes vs. nonchain small strokes around the nose; Phase 3 strokes vs. nonchain large strokes over the eyes). This supports the idea that these neurons were not simply coding movement form or amplitude.

Most changes coding sequence initiation were excitatory. Because reticulata activation via the direct pathway should be inhibitory, our results suggest the sequence coding signal arises via excitatory activation from the subthalamic nucleus (Nakanishi et al., 1987) through the indirect (Albin et al., 1989) or corticosubthalamic pathway (Kitai \& Deniau, 1981; Fujimoto \& Kita, 1993; Kolomiets et al., 2001). Excitatory subthalamic inputs through cortical pathways may predominate over inhibitory striatal input in this instance to excite central SNpr (Mink, 1996). The vital role of subthalamus in 
controlling information flow through the basal ganglia is also supported by observations that human Parkinsonian symptoms are reversed by high frequency stimulation in subthalamus (Limousin et al., 1995).

The timing of activation of SNpr neurons at the initiation of this behavioural sequence also makes it unlikely that a direct striatal projection is the main contributing factor. Neurons in the dorsolateral neostriatum have been found to fire throughout the entire sequence, including later phases (Aldridge \& Berridge, 1998). If direct inhibitory GABAergic control by the striatum was in control, one might expect SNpr neurons to be inhibited throughout syntactic chains instead of excited in early phases only. Our findings suggest that excitatory subthalamic inputs relay sequence initiation signals to $\mathrm{SNpr}$, perhaps embedded in loops of ascending flow of information through the basal ganglia (Haber et al., 2000).

Our findings support the idea that the basal ganglia focus or select certain movements by dynamically inhibiting competing motor mechanisms (Mink, 1996). Our findings show that, during the chain grooming sequence, there is a 'suppression of excitation' of activation during Phases 2 and 3 in comparison to the activation produced by these same movements during nonchain grooming. Thus, the executive control mechanisms for this grooming sequence may be mediated in SNpr by both fast excitatory connections from the subthalamic nucleus and slower diffuse inhibition from the striatum. This inhibition does not eliminate SNpr activity in the classic sense of absolute inhibition, but instead produces a 'relative suppression' that may represent the dynamic circuit organization necessary to focus and execute the ongoing grooming sequence.

Because neurons in the reticulata fire at sequence onset for syntactic grooming chains, they may be especially tuned to the initiation of this sequential pattern of grooming via subthalamic projections. By contrast, neurons in the dorsolateral neostriatum seem to be more involved in coding the implementation of the later phases of the pattern as well (Cromwell \& Berridge, 1996; Aldridge \& Berridge, 1998; Berridge \& Aldridge, 2000a; Berridge \& Aldridge, 2000b).

Close inspection of the timing indicates that SNpr firing bursts may be a consequence or efferent copy marker for the behavioural initiation of sequences, rather than a preceding cause of initiation. The strongest activation began after the first forward limb movement had already started at the beginning of the first Phase 1 ellipse stroke. In other words, the greatest neuronal activation in the reticulata coincides with the onset of the chain as it emerges from less sequentially stereotyped movements. This timing suggests that SNpr activation may have an important role in signalling other structures involved in implementation that the specific serial pattern has begun. Because syntactic chains are embedded in longer bouts of grooming, these neuronal responses mark the specific onset of syntactic sequences as a serial pattern and do not simply signal the onset of grooming itself. By contrast, suppression in firing rate occurred immediately before the initiation of syntactic grooming chains. An anticipatory position before the sequence makes that early depression a more plausible candidate for a role either in causing or in preparing for the ensuing sequential pattern.

Does SNpr encode sequences in general? Our present data indicate only that they code the particular sequential pattern of syntactic grooming chains. That is consistent with a more general role in sequential coding, but investigation of a general role in other behavioural sequences would require future experiments. However, it has been noted that general sequential deficits involving both actions and words can occur in Parkinson's disease (Martin et al., 1994; Marsden \& Obeso, 1994), and that normal SNpr activity is disrupted in an animal model of that disease (Wichmann et al., 1999). Our findings are consistent with the notion that altered output of SNpr may disrupt initiation and processing of lawful behavioural sequences in Parkinson's disease. Conversely, different changes in $\mathrm{SNpr}$ neuronal coding might contribute to excessively strong initiation of sequential patterns of behaviour in other basal ganglia diseases, such as Tourette's syndrome or obsessive-compulsive disorder.

\section{Acknowledgements}

This work was supported by National Institutes of Health Grants NS31650. We thank Rick Kindt for the computing assistance and Nadia Siddiqui for technical assistance. T. Allison, E. Anderson, R. Arora, L. Dunlop, L. Hadden, I. Hui, A. Kadushin, A. Kim, S. Kulkarni, J. Newbern, E. Okoro, L. Pierchala, L. Rogers, A. Rosen, C. Sauber, L. Shikhman, S. Srinidhi, T. Wang and M. Whitney assisted with video scoring.

\section{Abbreviations}

SNpr, substantia nigra pars reticulata.

\section{References}

Albin, R.L., Young, A.B. \& Penney, J.B. (1989) The functional anatomy of basal ganglia disorders. Trends Neurosci., 12, 366-375.

Aldridge, J.W. \& Berridge, K.C. (1998) Coding of serial order by neostriatal neurons: a 'natural action' approach to movement sequence. J. Neurosci., 18, 2777-2787.

Aldridge, J.W., Berridge, K.C., Herman, M. \& Zimmer, L. (1993) Neuronal coding of serial order: syntax of grooming in the neostriatum. Psychol. Sci., 4, 391-395.

Beiser, D.G. \& Houk, J.C. (1998) Model of cortical-basal ganglionic processing: encoding the serial order of sensory events. J. Neurophysiol., 79, 3168-3188.

Berns, G.S. \& Sejnowski, T.J. (1998) A computational model of how the basal ganglia produce sequences. J. Cogn. Neurosci., 10, 108-121.

Berridge, K.C. \& Aldridge, J.W. (2000a) Super-stereotypy I: Enhancement of a complex movement sequence by systemic dopamine D1 agonists. Synapse, 37, 194-204.

Berridge, K.C. \& Aldridge, J.W. (2000b) Super-stereotypy II: Enhancement of a complex movement sequence by intraventricular dopamine D1 agonists. Synapse, 37, 205-215.

Berridge, K.C., Fentress, J.C. \& Parr, H. (1987) Natural syntax rules control action sequence of rats. Behav. Brain Res., 23, 59-68.

Berridge, K.C. \& Whishaw, I.Q. (1992) Cortex, striatum, and cerebellum: control of serial order in a grooming sequence. Exp. Brain. Res., 90, 275290.

Churchward, P.R., Butler, E.G., Finkelstein, D.I., Aumann, T.D., Sudbury, A., Horne \& M.K. (1997) A comparison of methods used to detect changes in neuronal discharge patterns. J. Neurosci. Meth, 76, 203-210.

Cromwell, H.C. \& Berridge, K.C. (1996) Implementation of action sequences by a neostriatal site: a lesion mapping study of grooming syntax. $J$. Neurosci., 16, 3444-3458.

Curra, A., Berardelli, A., Agostino, R., Modugno, N., Puorger, C.C., Accornero, N. \& Manfredi, M. (1997) Performance of sequential arm movements with and without advance knowledge of motor pathways in Parkinson's disease. Mov. Disord., 12, 646-654.

DeLong, M.R., Crutcher, M.D. \& Georgopoulos, A.P. (1983) Relations between movement and single cell discharge in the substantia nigra of the behaving monkey. J. Neurosci., 3, 1599-1606.

Fujimoto, K. \& Kita, H. (1993) Response characteristics of subthalamic neurons to the stimulation of the sensorimotor cortex in the rat. Brain Res., 609, $185-192$.

Gulley, J.M., Kuwajima, M., Mayhill, E. \& Rebec, G.V. (1999) Behaviorrelated changes in the activity of substantia nigra pars reticulata neurons in freely moving rats. Brain Res., 845, 68-76.

Haber, S.N., Fudge, J.L. \& McFarland, N.R. (2000) Striatonigrostriatal pathways in primates form an ascending spiral from the shell to the dorsolateral striatum. J. Neurosci., 20, 2369-2382.

Harrington, D.L. \& Haaland, K.Y. (1991) Sequencing in Parkinson's disease - 
abnormalities in programming and controlling movement. Brain, 114, $99-115$.

Ho, A.K., Bradshaw, J.L., Cunnington, R., Phillips, J.G. \& Iansek, R. (1998) Sequence heterogeneity in Parkinsonian speech. Brain Language, 64, 122145 .

Jaeger, D., Gilman, S. \& Aldridge, J.W. (1990) A multiwire microelectrode for single unit recording in deep brain structures. J. Neurosci. Meth, 32, 143148.

Kermadi, I. \& Joseph, J.P. (1995) Activity in the caudate nucleus of monkey during spatial sequencing. J. Neurophysiol., 74, 911-933.

Kitai, S.T. \& Deniau, J.M. (1981) Cortical inputs to the subthalamus: intracellular analysis. Brain Res., 214, 411-415.

Kolomiets, B.P., Deniau, J.M., Mailly, P., Menetrey, A., Glowinski, J. \& Thierry, A.M. (2001) Segregation and convergence of information flow through the cortico-subthalamic pathways. J. Neurosci., 21, 5764-5772.

Lang, A.E., Consky, E. \& Sandor, P. (1993) 'Signing tics' - insights into the pathophysiology of symptoms in Tourette's syndrome. Ann. Neurol., 33, 212-215.

Lashley, K.S. (1951) The problem of serial order in behavior. In Jeffress, L.A (ed.), Cerebral Mechanisms in Behavior. Wiley, New York, pp. 112-146.

Levy, R., Hazrati, L.N., Herrero, M.T., Vila, M., Hassani, O.K., Mouroux, M., Ruberg, M., Asensi, H., Agid, Y., Feger, J., Obeso, J.A., Parent, A. \& Hirsch, E.C. (1997) Re-evaluation of the functional anatomy of the basal ganglia in normal and parkinsonian states. Neuroscience, 76, 335-343.

Lieberman, P. (2001) Human language and our reptilian brain. The subcortical bases of speech, syntax, and thought. Perspect. Biol. Med., 44, 32-51.

Lieberman, P., Kako, E., Friedman, J., Tajchman, G., Feldman, L.S. \& Jiminez, E.B. (1992) Speech production, syntax comprehension, and cognitive deficits in Parkinson's disease. Brain Language, 43, 169-189.

Limousin, P., Pollak, P., Benazzouz, A., Hoffmann, D., Le Bas, J.F., Broussolle, E., Perret, J.E. \& Benabid, A.L. (1995) Effect of parkinsonian signs and symptoms of bilateral subthalamic nucleus stimulation. Lancet, 345, 91-95.

Mahon, S., Delord, B., Deniau, J.M. \& Charpier, S. (2000) Intrinsic properties of rat striatal output neurones and time-dependent facilitation of cortical inputs in vivo. J. Physiol. (Lond.), 527, 345-354.

Marsden, C.D. \& Obeso, J.A. (1994) The functions of the basal ganglia and the paradox of stereotaxic surgery in Parkinson's disease. Brain, 117, 877-897.

Martin, K.E., Phillips, J.G., Iansek, R. \& Bradshaw, J.L. (1994) Inaccuracy and instability of sequential movements in Parkinson's disease. Exp. Brain Res., 102, 131-140.

Matsumoto, N., Hanakawa, T., Maki, S., Graybiel, A.M. \& Kimura, M. (1999) Nigrostriatal dopamine system in learning to perform sequential motor tasks in a predictive manner. J. Neurophysiol., 82, 978-998.
Mink, J.W. (1996) The basal ganglia: focused selection and inhibition of competing motor programs. Prog. Neurobiol., 50, 381-425.

Mushiake, H. \& Strick, P.L. (1995) Pallidal neuron activity during sequential arm movements. J. Neurophysiol., 74, 2754-2758.

Nakanishi, H., Kita, H. \& Kitai, S.T. (1987) Intracellular study of rat substantia nigra pars reticulata neurons in an in vitro slice preparation: electrical membrane properties and response characteristics to subthalamic stimulation. Brain Res., 437, 45-55.

O'Quinn, A.N. \& Thompson, R.J. Jr (1980) Tourette's syndrome: an expanded view. Pediatrics, 66, 420-424.

Rapoport, J.L. \& Wise, S.P. (1988) Obsessive-compulsive disorder: evidence for basal ganglia dysfunction. Psychopharmacol. Bull., 24, 380-384.

Redgrave, P., Prescott, T.J. \& Gurney, K. (1999) The basal ganglia: a vertebrate solution to the selection problem? Neuroscience, 89, 1009-1023.

Schultz, W. (1986a) Activity of pars reticulata neurons of monkey substantia nigra in relation to motor, sensory, and complex events. J. Neurophysiol., 55, 660-677.

Schultz, W. (1986b) Responses of midbrain dopamine neurons to behavioral trigger stimuli in the monkey. J. Neurophysiol., 56, 1439-1461.

Schultz, W. (1998) Predictive reward signal of dopamine neurons. $J$. Neurophysiol., 80, 1-27.

Smith, Y., Bevan, M.D., Shink, E. \& Bolam, J.P. (1998) Microcircuitry of the direct and indirect pathways of the basal ganglia. Neuroscience, 86, 353387.

Swanson, L.W. (1992) Brain Maps: Structure of the Rat Brain. Elsevier, Amsterdam

Toates, F. (1990) Obsessional Thoughts and Behaviour. Thorsons Publishing Group, Wellingborough.

Van Spaendonck, K.P., Horstink, M.W.I.M., Buytenhuijs, E.L., Cools, A.R. \& Berger, H.J.C. (1996) Executive functions and disease characteristics in Parkinson's disease. Neuropsychologia, 34, 617-626.

Waddington, J.L., Deveney, A.M., Clifford, J., Tighe, O., Croke, D.T., Sibley, D.R. \& Drago, J. (1998) Behavioral analysis of multiple D1-like dopamine receptor subtypes: new agents and studies in transgenic mice with D1A receptor knockout. Adv. Pharmacol. (New York), 42, 514-517.

Westwater, H., McDowall, J., Siegert, R., Mossman, S. \& Abernethy, D. (1998) Implicit learning in Parkinson's disease: evidence from a verbal version of the serial reaction time task. J. Clin. Exp. Neuropsychol., 20, 413-418.

Wichmann, T., Bergman, H., Starr, P.A., Subramanian, T., Watts, R.L. \& DeLong, M.R. (1999) Comparison of MPTP-induced changes in spontaneous neuronal discharge in the internal pallidal segment and in the substantia nigra pars reticulata in primates. Exp. Brain Res., 125, 397-409.

Wichmann, T. \& DeLong, M.R. (1996) Functional and pathophysiological models of the basal ganglia. Curr. Opin. Neurobiol., 6, 751-758. 